\title{
Spin-flip processes and ultrafast magnetization dynamics in Co - unifying the microscopic and macroscopic view of femtosecond magnetism
}

\author{
M. Cinchetti* M. Sánchez Albaneda, D. Hoffmann, T. Roth, J.-P. Wüstenberg, \\ M. Krauß, O. Andreyev, H. C. Schneider, M. Bauer, and M. Aeschlimann \\ University of Kaiserslautern, Physics Department, \\ Erwin-Schrödinger-Str. 46, 67663 Kaiserslautern, Germany
}

(Dated: July 9, 2021)

\begin{abstract}
The femtosecond magnetization dynamics of a thin cobalt film excited with ultrashort laser pulses has been studied using two complementary pump-probe techniques, namely spin-, energyand time-resolved photoemission and time-resolved magneto-optical Kerr effect. Combining the two methods it is possible to identify the microscopic electron spin-flip mechanisms responsible for the ultrafast macroscopic magnetization dynamics of the cobalt film. In particular, we show that electron-magnon excitation does not affect the overall magnetization even though it is an efficient spin-flip channel on the sub-200 fs timescale. Instead we find experimental evidence for the relevance of Elliott-Yafet type spin-flip processes for the ultrafast demagnetization taking place on a time scale of $300 \mathrm{fs}$.
\end{abstract}

PACS numbers: 75.70.Ak, 78.47.+p, 79.60.Dp 
Understanding the microscopic processes governing magnetization dynamics on the femtosecond time scale is one of the most challenging and interesting issues in today's condensed matter physics. There is nowadays much experimental evidence that the excitation of a ferromagnetic film with an ultrashort laser pulse gives rise to a demagnetization on a time scale of a few hundred femtoseconds 1, 2, 3, 4, 5, 6]. However, the microscopic processes responsible for these ultrafast changes in the macroscopic magnetism of the ferromagnet have not been unambiguously identified yet. A complete understanding of such processes would be extremely helpful for technical applications like ultrafast magnetization-switching schemes for read-write processes. Most of the recent experimental approaches applied in this field employed time-resolved magneto-optical strategies [1, 2, 4, 5, 6]. Such experiments access the ensemble spin dynamics taking place on the femtosecond time scale. On the other hand, spin- and time-resolved photoemission addresses the dynamics of single electron spinflip processes, which are responsible - from a microscopic point of view - for the observed changes in the sample magnetization. Bringing together these two experimental methods has, therefore, the potential for an efficient access to the microscopic view of the ultrafast dynamics of macroscopic magnetism.

In this letter such a complementary approach is presented for the first time and is used to obtain a more comprehensive understanding of the ultrafast magnetization dynamics of a thin cobalt film. Time-resolved magneto-optical Kerr effect (TR-MOKE), as well as spin-, energy- and time-resolved two-photon photoemission (SETR-2PPE) are used to address respectively the macroscopic and microscopic dynamical response of the ferromagnet induced by ultrashort optical excitation. SETR-2PPE probes the time evolution of the spin polarization of single optically excited electrons at a time-resolution down to a few femtoseconds. In comparison with TR-MOKE data we find that characteristic features in the observed complex time dependence of the SETR-2PPE signal can be directly correlated to changes in the magnetization of the sample. In this way, we are able to draw conclusions about the microscopic spin-flip mechanisms leading to the observed macroscopic ultrafast demagnetization of the thin Co film.

There are three main mechanisms through which an excited electron can flip its spin in a ferromagnetic metal: (i) a Stoner excitation (ii) an inelastic electron-spinwave scattering event (magnon excitation), or (iii) an Elliott-Yafet type of spin-flip scattering with impurities or phonons [7, 8]. Whereas for excitation energies above the vacuum energy 
( $E-E_{F}>\Phi, \Phi=$ work function), channels (i) and (ii) have been identified within static SPEELS measurements [9, 10], there exists no direct experimental access to optically excited electrons which are most relevant for laser induced ultrafast demagnetization. Thus, the excitation regime essential for ultrafast laser demagnetization $\left(E-E_{F}<\Phi\right)$ remains to be explored experimentally. Theoretically, short wavelength spinwave excitations have been studied in 11, 12]. According to these studies, spinwave scattering dominates Stoner excitation processes in the very low energy regime (roughly $\left(E-E_{F}\right)<3 \mathrm{eV}$ ). Moreover, first-principles GW and T-matrix calculations including spin-flip scattering [13] have shown that the coupling to spinwaves is an important inelastic decay channel of excited electrons in itinerant ferromagnets, especially for energies $\left(E-E_{F}\right)<1.5 \mathrm{eV}$. On the other hand, a detailed analysis of TR-MOKE experiments has provided some evidence that Elliott-Yafet processes have to be considered for a correct interpretation of femtosecond magnetization data 14]. In the following we will show that electron-magnon interaction indeed contributes significantly to the spin dynamics of single electrons excited close to the Fermi level $\left(E_{F}\right)$ in a Co thin film. However we also find that this process cannot account for the observed ultrafast demagnetization. Instead we identify clear support for the Elliott-Yafet scenario.

The detailed experimental setup for SETR-2PPE has been described elsewhere [15]. Briefly, we use the second harmonic of a femtosecond Ti:sapphire laser with photon energy $h \nu=3.1 \mathrm{eV}$. The beam is divided into two equally intense, collinear and orthogonally polarized beams with an adjustable time delay. The pump photons (here, s-polarized) excite electrons from their ground state into an unoccupied intermediate state. The delayed probe photons (p-polarized) excite the electrons above the vacuum level. The angle of incidence of the laser on the sample is $45^{\circ}$; the fluence of the pump and probe pulses is $3 \mu \mathrm{J} / \mathrm{cm}^{2}$. The photoemitted electrons are analyzed in normal emission geometry in an ultrahigh vacuum system equipped with a commercial cylindrical sector energy analyzer (Focus CSA 300) and a spin detector (Focus SPLEED).

Co films with a thickness of $10 \mathrm{~nm}$ are grown quasi-epitaxially on a $\mathrm{Cu}(001)$ substrate, and possess in-plane magnetization easy axis along the (110) direction of the $\mathrm{Cu}$ crystal. For the SETR-2PPE experiments the Co films are remanently magnetized by an external field applied along the easy axis direction. The spin detector measures the projection of the spin polarization along the easy axis: $P=\left(N^{+}-N^{-}\right) /\left(N^{+}+N^{-}\right)$, where $N^{+}\left(N^{-}\right)$is the number of detected majority (minority) electrons. 
After the SETR-2PPE experiments, the same Co film has been investigated ex-situ by TR-MOKE. A two-color pump-probe experiment has been used, where the pump pulses are generated by a multipass amplifier system operating at a central wavelength of $800 \mathrm{~nm}$ and delivering $50 \mathrm{fs}$ pulses with $1.6 \mathrm{~mJ} /$ pulse at a repetition rate of $1 \mathrm{kHz}$. The probe pulses are obtained by frequency doubling a small fraction $(\approx 1 / 10)$ of the pump beam in a $\beta$-bariumborate crystal. The bichromatic approach enables an efficient suppression of bleaching effects which may otherwise lead to a misinterpretation of the MOKE data 5]. The experiments were performed by recording, for every pump-probe delay, full hysteresis loops in longitudinal configuration. In this way the shape of the hysteresis can be used as a monitor of the sample quality during measurements. The external field vector is applied parallel to the in-plane easy axis direction. The inset of Figure 3 shows two exemplary hysteresis loops recorded respectively without the pump pulse (black curve) and $675 \mathrm{fs}$ after it (grey curve).

In a time- and energy-resolved 2PPE measurement the recorded quantity (crosscorrelation curve) is the number of excited electrons at a certain energy as a function of the time delay between the pump and the probe pulse (population decay). Figure 1 (a) and (b) show the cross-correlation curve of an intermediate state with an energy of $2.8 \mathrm{eV}$ and $0.4 \mathrm{eV}$ above $E_{F}$, respectively. In case the $2 \mathrm{PPE}$ signal is governed exclusively by an exponential population decay of the intermediate state, the experimental data can be fitted by a rate-equation model, where the lifetime $\tau$ of the selected intermediate level is the only fit parameter. Corresponding calculations to simulate our experimental data were performed using the formalism described in [16] in the limit of rapid dephasing between the electronic excitation and the driving laser field. The dashed lines in Figure 1 1 correspond to such a fit. Whereas the data recorded at $2.8 \mathrm{eV}$ intermediate energy (a) are almost perfectly described by this model, we find for the lower energy (b) rather significant deviations at temporal delays between 50 and $200 \mathrm{fs}$. In order to quantify these deviations, we evaluate the refilling $R=\left(Y_{m}-Y_{f}\right) /\left(Y_{m}+Y_{f}\right)$, where $Y_{m}$ is the measured 2PPE yield, and $Y_{f}$ the result of the rate-equation model. The obtained curves are shown on the right scale (open circles). Clearly, at $2.8 \mathrm{eV}$ intermediate energy $R$ is almost zero, while at $0.4 \mathrm{eV}$ it is significantly different from zero up to $200 \mathrm{fs}$ delay, and reaches its maximum value of $\sim 1 \%$ at $120 \mathrm{fs}$. The explanation for this additional contribution to the $2 \mathrm{PPE}$ signal is that secondary electrons scatter into the probed intermediate level and lead to a repopulation (refilling) of the state itself (see Figure $1(\mathrm{c})$ ). From the values of $R$ we can conclude that in our case refilling elec- 
trons constitute at maximum $1 \%$ of the electron population at the considered intermediate level. There are different relaxation mechanisms resulting in refilling electrons; the most relevant is the scattering of an optically excited (hot) electron in a higher excited state with a cold electron from below $E_{F}$. Further details on refilling can be found in [15].

Let us now concentrate on the time evolution of the spin polarization of the refilling electrons in the intermediate state located $0.4 \mathrm{eV}$ above $E_{F}$ as detected by SETR-2PPE. Our aim is to identify which kind of spin-flip scattering events take place during the decay process as schematically shown in Figure 1(c). Figure 2 shows the dependence of the inplane polarization of electrons in the intermediate state at this energy. In particular, we plot the normalized polarization changes, defined as $\widetilde{\Delta P}=\left(P-P_{\min }\right) /\left(P_{0}-P_{\min }\right)$, where $P_{0}$ is the polarization at zero delay and $P_{\min }$ the minimum of the polarization over the studied delay range. $\widetilde{\Delta P}$ exhibits a complex time-dependence with two maxima at $30 \mathrm{fs}$ and $120 \mathrm{fs}$, and two minima at $75 \mathrm{fs}$ and $350 \mathrm{fs}$.

To separate different contributions to the spin- and energy-resolved electron dynamics, we use a numerical model which phenomenologically includes carrier Boltzmann scattering together with carrier transitions due to pump and probe pulses. This model shows which features of the experimental results can be explained without the existence of magnetic correlations (such as magnon excitation). More precisely, we solve dynamical, energy-resolved equations for the electronic occupation $n_{s}(E)$ of single-particle states with spin $s$ and energy $E$ of the form

$$
\begin{aligned}
\frac{\partial}{\partial t} n_{s}(E)= & -\Gamma_{s}^{\mathrm{out}}(E) n_{s}(E)+\Gamma_{s}^{\mathrm{in}}(E)\left[1-n_{s}(E)\right] \\
& +\left.\frac{\partial}{\partial t} n_{s}(E)\right|_{\mathrm{pp}}
\end{aligned}
$$

where the last term on the right-hand side describes electronic transitions due to the pump and probe laser fields and includes the density of states which is taken from experiment. The in and out-scattering rates in Eq. (11) for weak nonequilibrium situations are determined from the measured spin-dependent electron lifetimes using $\Gamma_{s}^{\text {out }}(E)=-b(-E) / \tau_{s}(E)$, where $b(E)=[\exp (\beta E)-1]^{-1}$ is the Bose function, together with the detailed-balance relation $\Gamma_{s}^{\text {out }}(E)=\exp (\beta E) \Gamma_{s}^{\text {in }}(E)$. Using these relations implies that the dynamics obtained from Eq. (11) describes transitions involving scattering events with both minority and majority electrons, but does not account for the full correlated electron dynamics. In particular, 
our approach can describe the influence of the dynamical single-particle occupation on the experiment, which includes effects such as cascade electronic scattering events.

The result of the simulation for delays up to $200 \mathrm{fs}$ is shown in the inset of Figure2 (dashed line), together with the measured data. Clearly, a good agreement between experiment and calculations is achieved only for the first $100 \mathrm{fs}$. Here, the spin polarization first increases from its value at zero delay to a maximum close to $30 \mathrm{fs}$. This is due to the longer lifetime of majority electrons compared to the lifetime of minority electrons [17]. This difference implies that minority electrons depopulate the intermediate state at a faster rate than majority electrons, leading to a transient increase in the spin polarization (spin-filter effect). Between 30 and 75 fs the spin-filter effect starts to become irrelevant and the polarization decreases to the background value given by the spin-polarization excited by the pump and probe pulse separately. The successive increase of the spin polarization in the experiment, with a maximum around $120 \mathrm{fs}$, is not reproduced by the simulation. At this time delay we have a maximum of the refilling curve $R$ in Figure1(b). In order to interpret this behavior correctly, let us first observe that the calculations in [13] predict that minority electrons exhibit a higher cross-section for magnon excitation compared to majority electrons. Combining this results with the fact that our numerical simulation does not include magnon excitation, we can conclude that this process causes the measured increase of the spin polarization around 120 fs. In other words, the intermediate state at $0.4 \mathrm{eV}$ above $E_{F}$ is refilled by secondary electrons originating from energetically higher-lying minority electrons (with energy up to $3.1 \mathrm{eV}$ above $\left.E_{F}\right)$ : those electrons have a higher probability than majority electrons to scatter inelastically with a magnon and flip thereby their spin, turning into majority electrons and thus causing an increase in the measured spin polarization (see Figure 1 (c)).

We now discuss the behavior of the spin polarization for time delays between approximately 150 and $1000 \mathrm{fs}$. It shows a drop with a minimum around $350 \mathrm{fs}$ and a successive recovery. For such time delays, the number of pump laser excited electrons is so small that the observed behavior must be due to a change in the magnetic state of the electrons within the Fermi see. Indeed, after optical excitation the excited electrons and the produced secondary electrons thermalize to a hot Fermi-Dirac distribution on such a time scale. This implies that the feature observed in Figure 2 is related to a process in which the total magnetization is changing, and should be observed also with TR-MOKE. Figure 3 shows the TR-MOKE signal recorded for $10 \mathrm{~mJ} / \mathrm{cm}^{2}$ (triangles) and $2 \mathrm{~mJ} / \mathrm{cm}^{2}$ (squares) pump fluence. 
The curves are obtained by plotting, for a given pump-probe delay, the Kerr amplitude of the corresponding hysteresis loop, normalized by the value at zero delay. They show an ultrafast drop followed by a recovery. By reducing the pump fluence the minimum of the curve shifts to lower time delays. For the lower fluence the minimum is reached at approximately $400 \mathrm{fs}$, close to the minimum observed in the SETR-2PPE experiment. This fact gives a clear indication that the mechanism responsible for the reduction of the polarization in the SETR-2PPE experiment is the same one causing a reduction of the TR-MOKE signal, i.e. it is a process in which the total magnetization is changing. Koopmans et al. 14, 18] have argued that the ultrafast demagnetization observed in TR-MOKE experiments can be microscopically explained by assuming Elliott-Yafet type of spin-flip scattering of electrons with impurities or phonons. According to the same authors, such process leads to a decrease of the minimum of the TR-MOKE curves with the pump fluence. Such a behavior was indeed observed in [19], though, at lower fluencies and thereby with more subtle variations. Our TR-MOKE results give a valuable support to such interpretation and moreover, through the comparison with SETR-2PPE provide the experimental proof of the dominating role of Elliott-Yafet scattering in a microscopic description of ultrafast demagnetization.

In conclusion we have successfully combined two experimental techniques, one (TRMOKE) giving an insight into the ensemble femtosecond spin dynamics after laser excitation, the other (SETR-2PPE) providing additional information on the microscopic spin-flip processes responsible for the observed magnetization dynamics. We were able to conclude that even if microscopically spinwave excitation plays an important role in the ultrafast spin dependent electron dynamics, the ultrafast demagnetization of the studied thin Co film is mainly due to Elliott-Yafet type spin-flip processes.

Financial support from DFG priority program 1133 and the European research training network HPRN-CT-2002-00318 ULTRASWITCH is gratefully acknowledged. We thank F. Steeb for his support with the femtosecond laser system. One of us (M.C.) would like to thank R. Santachiara for stimulating discussions.

* cinchett@rhrk.uni-kl.de

[1] E. Beaurepaire, J. C. Merle, A. Daunois, and J. Y. Bigot, Phys. Rev. Lett. 76, 4250 (1996). 
[2] J. Hohlfeld, E. Matthias, R. Knorren, and K. H. Bennemann, Phys. Rev. Lett. 78, 4861 (1997).

[3] A. Scholl, L. Baumgarten, R. Jacquemin, and W. Eberhardt, Phys. Rev. Lett. 79, 5146 (1997).

[4] J. Güdde, U. Conrad, V. Jähnke, J. Hohlfeld, and E. Matthias, Phys. Rev. B 59, R6608 (1999).

[5] B. Koopmans, M. van Kampen, J. T. Kohlhepp, and W. J. M. de Jonge, Phys. Rev. Lett. 85, $844(2000)$.

[6] H. S. Rhie, H. A. Dürr, and W. Eberhardt, Phys. Rev. Lett. 90, 247201 (2003).

[7] R. Elliott, Phys. Rev. 96, 266 (1954).

[8] Y. Yafet, Solid State Physics, vol. 14 (Academic, New York, 1963).

[9] R. Vollmer, M. Etzkorn, A. Kumar, H. Ibach, and J. Kirschner, J. Magn. Magn. Mater. 272-276, 2126 (2004).

[10] M. Etzkorn, A. Kumar, R. Vollmer, H. Ibach, and J. Kirschner, Surf. Sci. 566-568, 241 (2004).

[11] M. Plihal and D.L. Mills, Phys. Rev. B 58, 14407 (1998).

[12] A.T. Costa, R.B. Muniz, and D.L. Mills, Phys. Rev. B 70, 054406 (2004).

[13] V.P. Zhukov, E.V. Chulkov, and P.M. Echenique, Phys. Rev. Lett. 93, 096401 (2004).

[14] B. Koopmans, J.J.M. Ruigrok, F. DallaLonga, and W.J.M. de Jonge, Phys. Rev. Lett. 95, 267207 (2005).

[15] R. Knorren, K. H. Bennemann, R. Burgermeister, and M. Aeschlimann, Phys. Rev. B 61, 9427 (2000).

[16] M. Bauer, S. Pawlik, and M. Aeschlimann, Phys. Rev. B 60, 5016 (1999).

[17] M. Aeschlimann, M. Bauer, S. Pawlik, W. Weber, R. Burgermeister, D. Oberli, and H. C. Siegmann, Phys. Rev. Lett. 79, 5158 (1997).

[18] B. Koopmans, H. Kicken, M. van Kampen, and W. de Jonge, J. Magn. Magn. Mater. 286, $271(2005)$.

[19] M. van Kampen, Ph.D. thesis, University of Eindhoven (2003). 


\section{FIGURES}

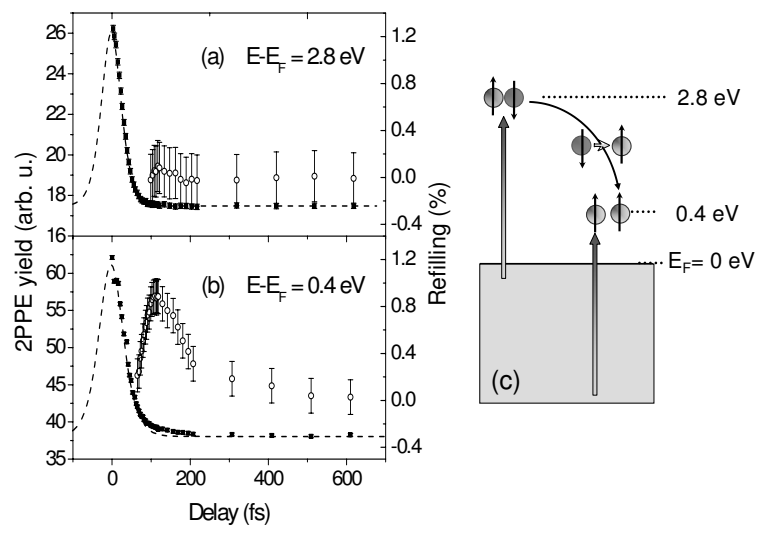

FIG. 1: M. Cinchetti, Physical Review Letters 


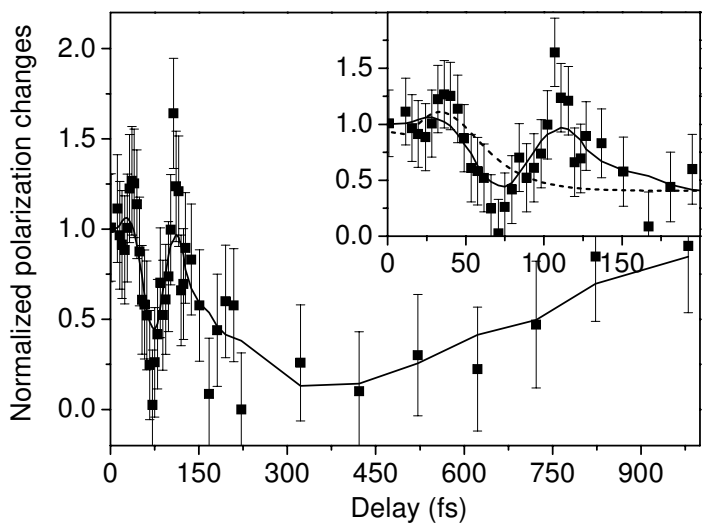

FIG. 2: M. Cinchetti, Physical Review Letters 


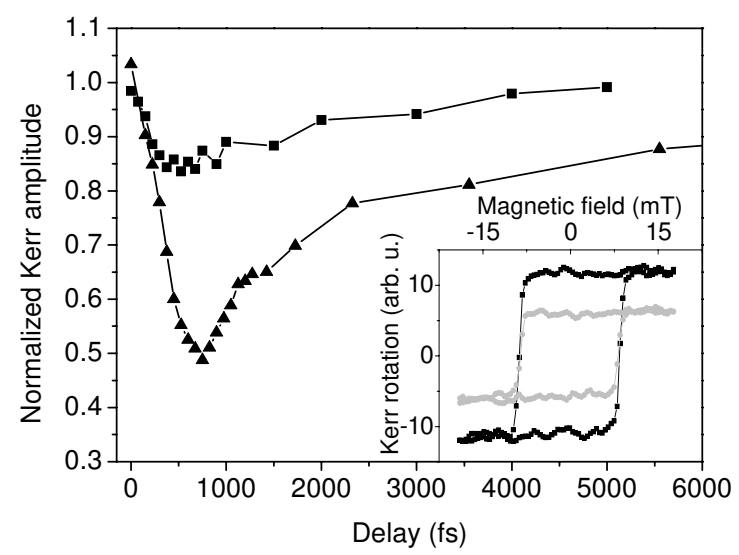

FIG. 3: M. Cinchetti, Physical Review Letters 


\section{CAPTIONS}

FIG. 1: (a) and (b). Left scale: 2PPE yield from an intermediate state with energy of $2.8 \mathrm{eV}$ (a) and $0.4 \mathrm{eV}$ (b) as a function of the delay time between the pump and the probe pulse. The fit of the experimental curves with a rate-equation model is plotted with dashed lines. Right scale: Refilling parameter $R$ as derived from the measured curves and the fitting curves.

(c) Schematic picture of a possible spin-dependent refilling process of the intermediate state at $0.4 \mathrm{eV}$, as discussed in the text.

FIG. 2: Time dependence of the spin polarization of electrons in the intermediate state at $0.4 \mathrm{eV}$ above $E_{F}$ as detected by SETR-2PPE. Inset: a zoomed picture of the same curve for time delays between $0 \mathrm{fs}$ and $200 \mathrm{fs}$ and the result of the numerical model discussed in the text (dashed line). The continuous line (obtained by smoothing the original data) is depicted as a guide to the eye for the reader.

FIG. 3: Results of TR-MOKE measurements on the same Co sample of Figure 2, taken with a pump fluence of $10 \mathrm{~mJ} / \mathrm{cm}^{2}$ (triangles) and $2 \mathrm{~mJ} / \mathrm{cm}^{2}$ (squares). Inset: two exemplary hysteresis loops recorded respectively without the pump pulse (black curve) and $675 \mathrm{fs}$ after it (grey curve). The pump power was $10 \mathrm{~mJ} / \mathrm{cm}^{2}$. 